\title{
Straddled numbers: numbers equal to the sum of powers of consecutive primes from the least prime factor to the largest prime factor
}

\author{
Miroslav Kureš \\ Department of Mathematics, Brno University of Technology \\ Technická 2, 61669 Brno, Czech Republic \\ e-mail: kures@fme.vutbr.cz
}

Received: 18 December 2018

Revised: 9 April 2019

Accepted: 12 April 2019

\begin{abstract}
Positive integers equal to the sum of powers of consecutive primes from the least prime factor to the largest prime factor are studied. They are called the straddled numbers and their properties are derived. There are also presented some findings of such numbers and asymptotic expansions are used, too.
\end{abstract}

Keywords: Sums of primes, Sums of prime powers, Waring-Goldbach-type problems.

2010 Mathematics Subject Classification: 11A51, 11P32.

\section{Introduction}

The whole story began around the turn of the millennium when Carlos Rivera [5] observed that 39 is a number with the following property:

(P1) A non-prime number equal to the sum of consecutive primes from the least prime factor to the largest prime factor.

Of course,

$$
39=3 \cdot 13=3+5+7+11+13 .
$$


It is not difficult to verify that 10,155 and 371 are other numbers with this property. Later (July 2000), Jud McCranie found the 5th number:

$$
2935561623745=5 \cdot 19 \cdot 53 \cdot 61 \cdot 9557877=5+7+11+\cdots+9557877 .
$$

On November 2002, Robert Munafo [4] discovered that

$454539357304421=3536123 \cdot 128541727=3536123+3536129+3536131+\cdots+128541727$.

So, six numbers with the property (P1) are known up to now. One can find them in the Online Encyclopedia of Integer Sequences under the code A055233 [7]. We change the property somewhat now:

(P2) A non-prime-square number equal to the sum of squares of consecutive primes from the least prime factor to the largest prime factor.

The smallest number with this property is 315797 . Indeed,

$$
315797=31 \cdot 61 \cdot 167=31^{2}+37^{2}+41^{2}+\ldots+167^{2} .
$$

The mersenneforum. org user, nicknamed alcall, has announced the finding of the second number with the (P2) property on September 3, 2018. It is the number 9634877:

$$
9634877=7 \cdot 41 \cdot 59 \cdot 569=7^{2}+11^{2}+13^{2}+\ldots+569^{2} .
$$

(Question A) Find other numbers with the property (P2).

We can continue.

(P3) A non-prime-cube number equal to the sum of cubes of consecutive primes from the least prime factor to the largest prime factor.

In this case, there is a nice solution: 160 .

$$
160=2^{5} \cdot 5=2^{3}+3^{3}+5^{3}
$$

(Question B) Find other numbers with the property (P3).

And finally:

(Question C) The first, second and third powers of primes can be generalized to any $m$-th power. Find also any numbers for $m>3$.

The Introduction was published as a puzzle on the authors's web page [3]. 


\section{The problem and some findings}

Let us consider a natural number $n$ with prime decomposition

$$
n=p_{1}^{k_{1}} \ldots p_{h}^{k_{h}} \quad\left(p_{1}<\cdots<p_{h} \text { primes, } h>1\right) .
$$

Let $P_{1}, \ldots, P_{r}$ be consecutive primes such that $P_{1}=p_{1}, P_{r}=p_{h}$ and let $m \in \mathbb{N}$. If $n$ satisfies

$$
n=P_{1}^{m}+\cdots+P_{r}^{m},
$$

we say that $n$ is a m-straddled number. It is clear that $n$ can not be simultaneously an $m_{1}$ straddled number and an $m_{2}$-straddled number for different $m_{1}, m_{2}$. Also, it seems also to be evident that infinitely many numbers are not $m$-straddled for any $m$. More precisely, we have:

Proposition 1. The cardinality of the set of natural numbers which are not $m$-straddled for any $m$ is $\aleph_{0}$.

Proof. We show that no number from the set $\left\{6^{u} ; u \in \mathbb{N}\right\}$ is $m$-straddled. If we assume

$$
2^{u} 3^{u}=2^{m}+3^{m}
$$

then there is an even number on the left-hand side and an odd number on the right-hand side which is impossible.

Now, we recapitulate our findings.

Proposition 2. 10, 39, 155, 371, 2935561623745 and 454539357304421 are 1-straddled numbers. 315797 and 9634877 are 2-straddled numbers. 160 is a 3-straddled number.

Proof. By direct verification.

In the expression

$$
n=p_{1}^{k_{1}} \ldots p_{h}^{k_{h}}=P_{1}^{m}+\cdots+P_{r}^{m}
$$

we observe:

Proposition 3. For every $m$-straddled number $n, r \geq h$ and $r$ is an odd number.

Proof. The assertion $r \geq h$ follows from the fact that $P_{1}=p_{1}, P_{h}=p_{r}$, where $p_{i}$ 's are primes while $P_{j}$ 's are consecutive primes. If $n$ is even, then $P_{1}=2$; thus, there is $r-1$ remaining $P_{j}$ 's and $r-1$ must me even for the sum to be even. If $n$ is odd, then the number of $P_{j}$ 's which is $r$ must me odd for the sum to be odd.

If we denote (as is usual) $\Omega=k_{1}+\cdots+k_{h}$, then the following assertion holds.

Proposition 4. For every $m$-straddled number $n, m<\Omega$ is satisfied.

Proof. Let us assume $m \geq \Omega$. Then

$$
p_{1}^{k_{1}} \ldots p_{h}^{k_{h}}=P_{1}^{k_{1}} p_{2}^{k_{2}} \ldots p_{h-1}^{k_{h-1}} P_{r}^{k_{h}}<P_{r}^{\Omega}<P_{1}^{m}+\cdots+P_{r}^{m}
$$

as $P_{r}^{\Omega} \leq P_{r}^{m}$ and hence

$$
p_{1}^{k_{1}} \ldots p_{h}^{k_{h}} \neq P_{1}^{m}+\cdots+P_{r}^{m} .
$$


We do not know of any example where $r=h$. Is it possible? We observe:

Proposition 5. For every $m$-straddled number $n, r>h$ in the following cases:

(i) $h$ is even

(ii) $m=1$

(iii) $r=2$

Proof. (i) It was already proved that $r$ is always odd.

(ii) For different positive integers $\geq 2$, the product is always greater than the sum even for the case $k_{1}=\cdots=k_{h}=1$.

(iii) Let us assume that $r=h=2$, i.e.

$$
P_{1}^{k_{1}} P_{2}^{k_{2}}=P_{1}^{m}+P_{2}^{m} .
$$

But it means

$$
P_{2}^{m}=P_{1}\left(P_{1}^{k_{1}-1} P_{2}^{k_{2}}-P_{1}^{m-1}\right)
$$

and so $P_{2}^{m}$ is a multiple of $P_{1}$ and it is impossible.

However, it seems that there may exist an $m$-straddled number $n$ with $r=h=3$. We will explain our view on a special case. Let us examine whether it can happen that

$$
P_{1}^{m-1} P_{2} P_{3}=P_{1}^{m}+P_{2}^{m}+P_{3}^{m} .
$$

The left-hand side is a product of three numbers of powers $m-1,1$ and 1, so an $(m+1)$-st power, while the right-hand side is a sum of three $m$-powers, so an $m$-th power. In general, for sufficiently large numbers $P_{1}, P_{2}$ and $P_{3}$,

$$
P_{1}^{m-1} P_{2} P_{3}>P_{1}^{m}+P_{2}^{m}+P_{3}^{m}
$$

nevertheless, there may be a finite number of exceptions. For $m=2$, there is only one triple of consecutive primes satisfying the reverse inequality, namely

$$
2 \cdot 3 \cdot 5<2^{2}+3^{2}+5^{2}
$$

for $m=3$, there are two such triples of consecutive primes

$$
\begin{aligned}
& 2^{2} \cdot 3 \cdot 5<2^{3}+3^{3}+5^{3} \\
& 3^{2} \cdot 5 \cdot 7<3^{3}+5^{3}+7^{3}
\end{aligned}
$$

and, e.g. for $m=500$, there are 111 such triples of consecutive primes, for last of them

$$
607^{499} \cdot 613 \cdot 617<607^{500}+613^{500}+617^{500}
$$

is still fulfilled. Therefore, the exact equality perhaps could happen for a great $m$, but we have not found it yet. 


\section{Asymptotic expansions}

For $x \in \mathbb{R}$, let $\pi(x)$ denote the number of primes $\leq x$. Further, we use $s_{k}^{m}$ for the sum of $m$-powers of first $k$ primes $\left(m \in \mathbb{N}, k \in \mathbb{N}_{0}, s_{0}^{m}=0\right.$ ). Then

$$
P_{1}^{m}+\cdots+P_{r}^{m}=s_{\pi\left(P_{r}\right)}^{m}-s_{\pi\left(P_{1}\right)-1}^{m} .
$$

There are known asymptotic formulae for $s_{k}^{m}$, in particular:

$$
\begin{aligned}
& s_{k}^{m} \sim \frac{k^{m+1}}{m+1} \log ^{m} k \quad \text { (Šalát and Znám, 1968, [6]) } \\
& s_{k}^{m} \sim \frac{k^{m+1}}{m+1} \log ^{m} k+\frac{m}{m+1} k^{m+1} \log ^{m} k \frac{\log \log k}{\log k} \quad \text { (Jakimczuk, 2014, [1]) }
\end{aligned}
$$

A comparison of these estimates and exact values is illustrated in Figure 1 and Figure 2.

\section{A note regarding the Waring-Goldbach problem: Additive prime number theory}

Our problem is not covered by any known theory. Nevertheless, the Waring-Goldbach problem is perhaps appropriate to remember.

Let $k$ be a natural number and let $A_{k}=\left\{0^{k}, 1^{k}, 2^{k}, 3^{k}, \ldots\right\}$ (powers of naturals including zero), $P_{k}=\left\{2^{k}, 3^{k}, 5^{k}, 7^{k}, \ldots\right\}$ (powers of primes).

Waring's problem: for a given $k$, to determine the least $h$, for which every natural number $n$ is expressible in a form $n=a_{1}+\ldots+a_{h}, a_{i} \in A_{k}$.

Waring-Goldbach problem: for a given $k$, to determine the least $H$, for which every sufficiently large natural number $n$ is expressible in a form $n=p_{1}+\ldots+p_{H}, p_{i} \in P_{k}$.

Results:

$\mathrm{k}=1$ :

$h=1$ (trivial)

$H=3$ (Goldbach's weak conjecture, proved probably by Harald Helfgott in 2013; for even $n$ it seems that $H=2$ which is Goldbach's conjecture)

$\mathrm{k}=2$ :

$h=4$ (Lagrange's four-square theorem known already to Diophantus)

$H \leq 5$
$\mathrm{k}=3:$

$h=9$ (Wieferich 1909)

$H \leq 9$

Results for higher $k$ are also known. See e.g. Angel Kumchev, [2].

Furthermore, some of the programmers' competitions are interesting. For example, there was the following question in the Project Euler, [8], No. 50: Which prime, below one-million, can be written as the sum of the most consecutive primes? The answer is that the largest prime below 1000000 written as the sum consecutive primes is 997651 which consists of 543 primes. 


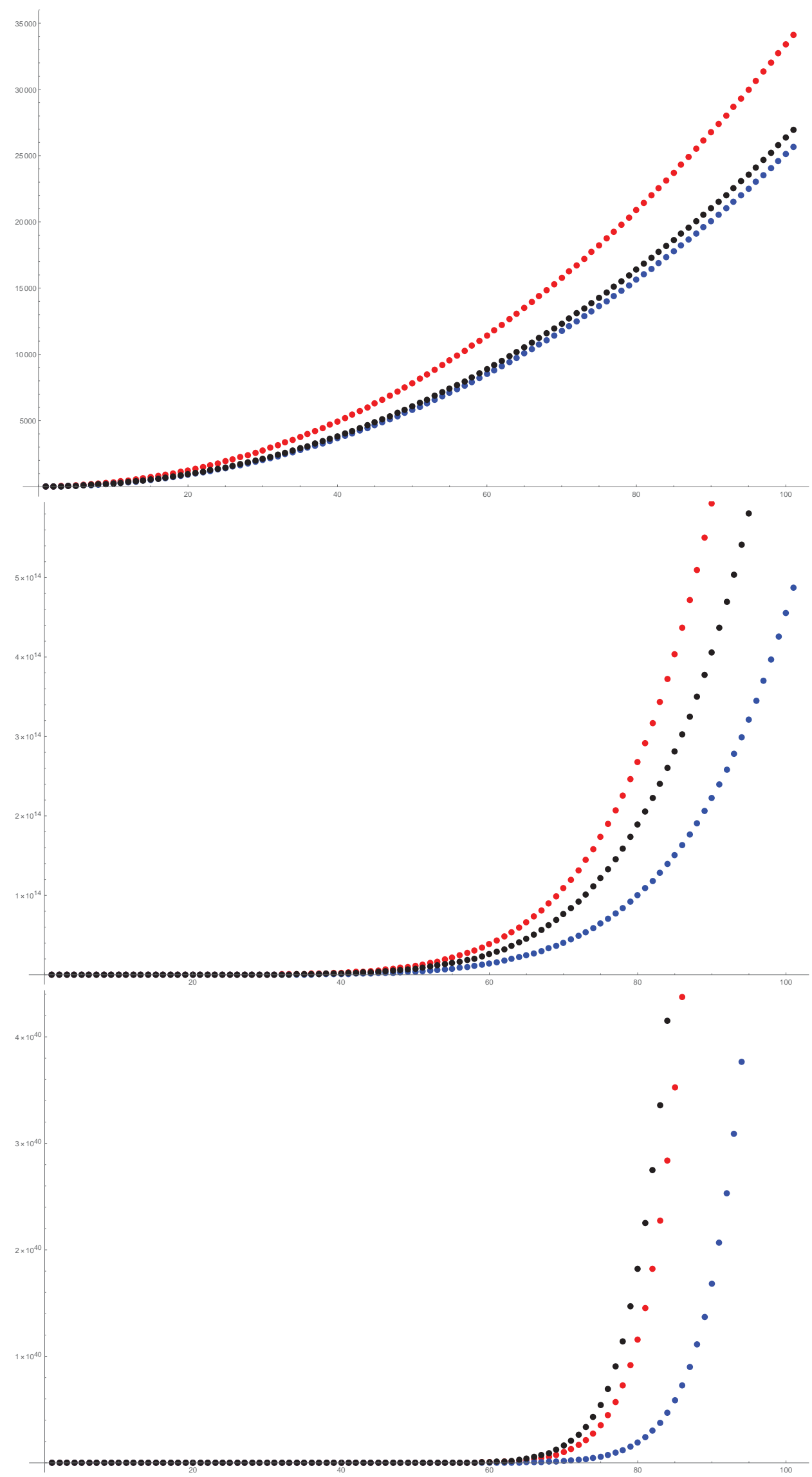

Figure 1. 100 primes starting from 7; 1st, 5th and 15th powers. Black: exact values; blue: Šalát, Znám; red: Jakimczuk. 


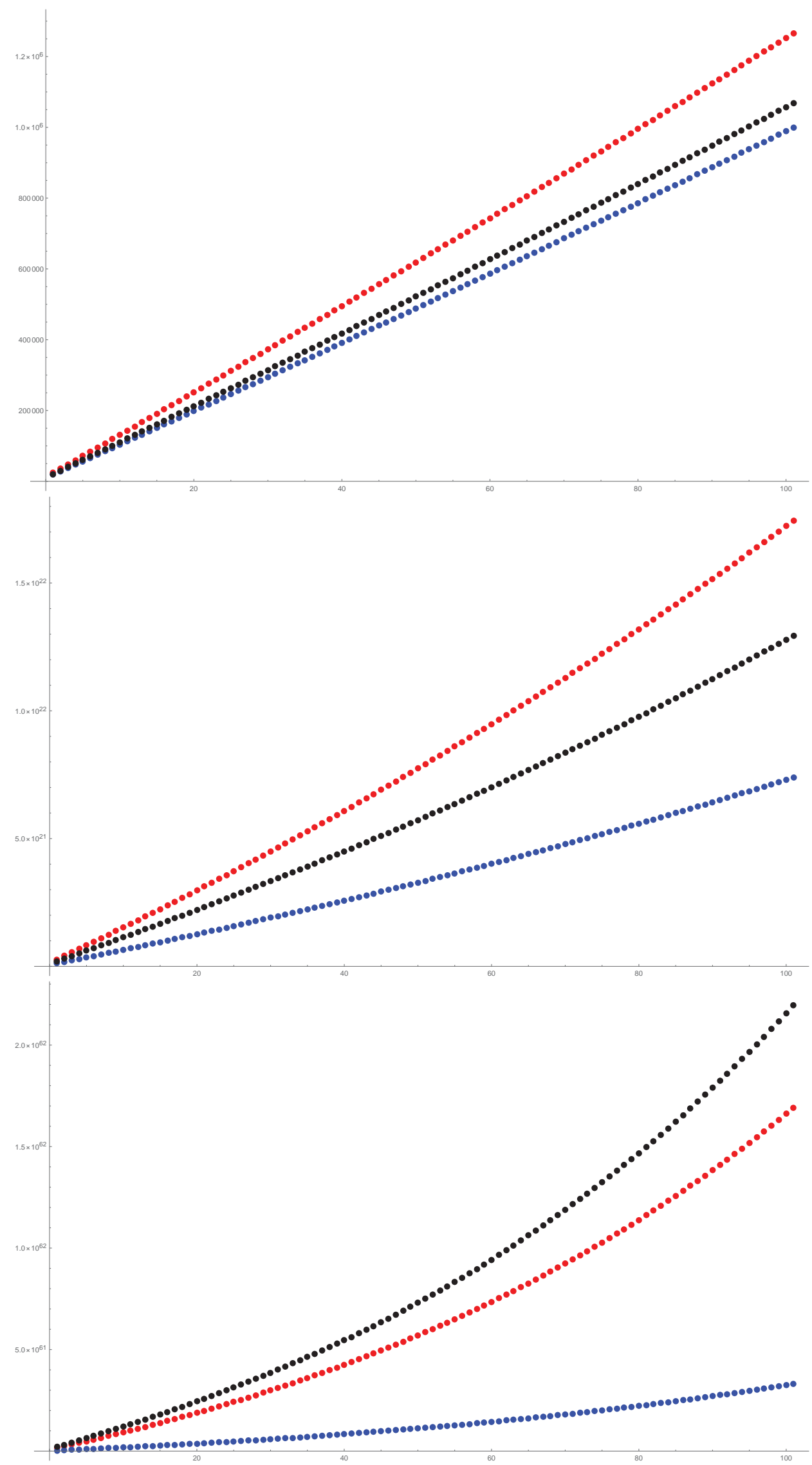

Figure 2. 100 primes starting from 10007; 1st, 5th and 15th powers. Black: exact values; blue: Šalát, Znám; red: Jakimczuk. 


\section{Acknowledgements}

The research has been supported by Brno University of Technology, the specific research plan being No. FSI-S-17-4464.

\section{References}

[1] Jakimczuk, R. (2014). Sums of Primes. An Asymptotic Expansion, International Journal of Contemporary Mathematical Sciences, 9 (16), 761-765.

[2] Kumchev, A. (2005). On the Waring-Goldbach problem for seventh powers, Proceedings of the American Mathematical Society, 133 (10), 2927-2937.

[3] Kureš, M. (2018). Puzzle 1: Sum of Powers of Primes. Available online: http:// searchfornumbers.blogspot.com/2018/08/puzzle-1-sum-of-powersof-primes.html. Accessed: April 9, 2019.

[4] Munafo, R. Notable Properties of Specific Numbers. Available online: https: / /mrob. com/pub/math/numbers-17.html. Accessed: April 9, 2019.

[5] Rivera, C. Puzzle 98. Curio 39. Available online: https://www.primepuzzles. net/puzzles/puzz_098.htm. Accessed: April 9, 2019.

[6] Šalát, T., \& Znám, Š. (1968). On sums of the prime powers, Acta Fac. Rer. Nat. Univ. Com. Math., 21, 21-24.

[7] Sloane, N. J. A. A055233. Composite numbers equal to the sum of the primes from their smallest prime factor to their largest prime factor. Online Encyclopedia of Integer Sequences. Available online: https: / / oeis . org/A055233. Accessed: April 9, 2019.

[8] Problem 50: Consecutive prime sum. Project Euler.net. Available online: https:// projecteuler. net/problem=50. Accessed: April 9, 2019. 